\title{
The Long-Term Evolutionary History of Gradual Reduction of CpG Dinucleotides in the SARS-CoV-2 Lineage
}

\author{
Sankar Subramanian
}

Citation: Subramanian, $\mathrm{S}$. The Long-Term Evolutionary History of Gradual Reduction of $\mathrm{CpG}$ Dinucleotides in the SARS-CoV-2 Lineage. Biology 2021, 10, 52. https://doi.org/10.3390/biology 10010052

Received: 30 November 2020 Accepted: 9 January 2021 Published: 12 January 2021

Publisher's Note: MDPI stays neutral with regard to jurisdictional clai$\mathrm{ms}$ in published maps and institutional affiliations.

Copyright: (C) 2021 by the author. Licensee MDPI, Basel, Switzerland. This article is an open access article distributed under the terms and conditions of the Creative Commons Attribution (CC BY) license (https:// creativecommons.org/licenses/by/ $4.0 /)$.
GeneCology Centre, School of Science and Engineering, University of the Sunshine Coast, Moreton Bay, QLD 4502, Australia; ssankara@usc.edu.au

Simple Summary: Severe acute respiratory syndrome coronavirus 2 (SARS-CoV-2) caused the coronavirus disease 2019 (COVID-19), a pandemic that infected over 81 million people worldwide. This has led the scientific community to characterize the genome of this virus, including its nucleotide composition. Investigation of the dinucleotide frequency revealed that the proportion of CG dinucleotides $(\mathrm{CpG})$ is highly reduced in the viral genomes. Since $\mathrm{CpG}$ dinucleotides is the target site for the host antiviral zinc finger protein, it has been suggested that the reduction in the proportion of $\mathrm{CpG}$ is the viral response to escape from the host defense machinery. In the present study, we investigated the time of origin of reduction in the CpG content. Whole genome analyses based on all representative viral genomes of the group Betacoronavirus revealed that the $\mathrm{CpG}$ content in the lineage of SARS-CoV-2 has been progressively declining over the past 1213 years. The depletion of CpG was found to occur at neutral-as well as selectively constrained-positions of the viral genomes.

\begin{abstract}
Recent studies suggested that the fraction of CG dinucleotides (CpG) is severely reduced in the genome of severe acute respiratory syndrome coronavirus 2 (SARS-CoV-2). The CpG deficiency was predicted to be the adaptive response of the virus to evade degradation of the viral RNA by the antiviral zinc finger protein that specifically binds to $\mathrm{CpG}$ nucleotides. By comparing all representative genomes belonging to the genus Betacoronavirus, this study examined the potential time of origin of $\mathrm{CpG}$ depletion. The results of this investigation revealed a highly significant correlation between the proportions of $\mathrm{CpG}$ nucleotide (CpG content) of the betacoronavirus species and their times of divergence from SARS-CoV-2. Species that are distantly related to SARS-CoV-2 had much higher CpG contents than that of SARS-CoV-2. Conversely, closely related species had low $\mathrm{CpG}$ contents that are similar to or slightly higher than that of SARS-CoV-2. These results suggest a systematic and continuous reduction in the CpG content in the SARS-CoV-2 lineage that might have started since the Sarbecovirus + Hibecovirus clade separated from Nobecovirus, which was estimated to be 1213 years ago. This depletion was not found to be mediated by the GC contents of the genomes. Our results also showed that the depletion of CpG occurred at neutral positions of the genome as well as those under selection. The latter is evident from the progressive reduction in the proportion of arginine amino acid (coded by CpG dinucleotides) in the SARS-CoV-2 lineage over time. The results of this study suggest that shedding $\mathrm{CpG}$ nucleotides from their genome is a continuing process in this viral lineage, potentially to escape from their host defense mechanisms.
\end{abstract}

Keywords: CpG dinucleotide; COVID-19; SARS-CoV-2; host defense; virus evolution; adaptation; zinc finger protein; Sarbecovirus

\section{Introduction}

Severe acute respiratory syndrome coronavirus 2 (SARS-CoV-2), which belongs to Betacoronaviruses, resulted in a pandemic that inflicted respiratory illness in human populations around the globe [1-8]. The outbreak caused by SARS-CoV-2 resulted in $>81$ million cases and $>1.7$ million deaths to date. A number of previous studies have characterized the nucleotide composition of the genome of SARS-CoV-2 [9-16]. These studies observed 
a much high proportion of $\mathrm{U}$ nucleotides and concomitant low fraction of $\mathrm{C}$ nucleotides in the genome $[12,14,15]$. The reason for such a discrepancy was attributed to a higher rate of $\mathrm{C} \rightarrow \mathrm{U}$ mutations compared to other mutations in the genome. Furthermore, the enzyme APOBEC3G in the viral hosts is known to deaminate cytosine to uracil nucleotides, particularly those present in the single-stranded RNA $[14,15,17]$. Investigating the dinucleotide patterns of SARS-CoV-2 genomes revealed that the $\mathrm{CpG}$ dinucleotides were severely reduced in these viral genomes [14,17-20]. Zinc finger antiviral protein (ZAP) protects cells from viral infections and this protein identifies viral genomes based on their CpG dinucleotide content (CpG content) [21-23]. Therefore, it has been suggested that the low CpG content of SARS-CoV-2 could have evolved to evade identification by ZAP [17].

Although previous studies have characterized the base composition of various viruses belonging to Betacoronaviruses, the pattern of evolution of CpG content in the SARS-CoV-2 genomes is unknown. For instance, it is unclear when $\mathrm{CpG}$ nucleotides started declining in the lineage of this SARS-CoV-2 and reached the level that we observe in their genomes today. This is important because understanding the evolutionary trajectory of base composition will help predict the future patterns of evolutionary changes. This will eventually assist in designing vaccines that will be effective, not only on the current strains, but also those that evolve in the immediate future. Hence, in the present study, we performed comparative genomic analyses using all representative genomes belonging to the genus Betacoronavirus to determine the pattern of evolution of $\mathrm{CPG}$ content over time.

\section{Materials and Methods}

\subsection{Genome Data}

Using the Taxonomy Browser application of the National Center for Biotechnology Information (NCBI), GenBank (https:/ / www.ncbi.nlm.nih.gov/Taxonomy/Browser/ wwwtax.cgi), we downloaded the complete genome sequences of belonging to the genus Betacoronavirus. We selected all representative genomes from the species belonging to the subgenus Embecovirus, Hibecovirus, Merbecovirus, and Sarbecovirus. This resulted in 79 complete betacoronavirus genomes, including those infecting human, bat, cow, civet, camel, dog, pangolins, pig, rabbit, rat, and yak (Supplementary Table S1). Using GenBank annotations, coding sequence (CDS) regions were extracted for the protein coding genes. Since all mutations at third codon positions of eight amino acids (alanine, arginine, glycine, leucine, proline, serine, threonine, and valine) do not change, the amino acids coded by the respective codons, these positions are considered as synonymous positions. For nonsynonymous positions, we extracted all second codon positions as any mutation in this position changes the amino acid coded by the codon. Excluding the codons UUA, UUG, CUA, CUG, CGA, CGG, AGA and AGG, first codon positions from all other codons are also extracted and considered as nonsynonymous positions. Mutation in the first codon position of the eight codons listed above does not change the amino acid coded by them and, hence, they were not considered as nonsynonymous positions.

\subsection{Phylogenetic Analysis}

We used the Maximum Likelihood (ML) method to infer the phylogenetic relationship among the viral genomes. This was accomplished by using the RaxML program [24]. The sophisticated General Time Reversible (GTR) method was used to model the evolution of nucleotides, as it addresses the differential rates of nucleotide changes, including transition/transversional and base compositional biases. To accommodate rate variations among nucleotide sites, we used the gamma model of evolution. To test the strength of phylogenetic relationships we used the bootstrap resampling procedure with 500 replications.

To compute evolutionary divergence, we first estimated the shape of the rate variation among site (gamma) using the program MEGA [25]. For this purpose, the ML method was used. The HKY model of nucleotide evolution and the discrete gamma model with five categories were used to compute the gamma value. This analysis produced a gamma value of 0.54 , which was used to estimate the pairwise evolutionary divergence using 
the Maximum Composite Likelihood method. To estimate the time of divergence, the Bayesian MCMC method-based software BEAST was used [26]. We opted for the HKY Gamma model for sequence evolution and Yule method for speciation. For calibrating and creating a linearized time tree, a clock rate of $1.1 \times 10^{-3}$ subs/site/year was used [27]. The program was run for 10 million MCMC generations by sampling every 1000 generations. This ensured an Effective Sample Size (ESS) of $>200$ for all parameters after excluding a 10\% burn-in (estimated by the program Tracer [28]). The results were combined from the sampled trees using the program TreeAnnotator (supplied as a part of the BEAST software) and the maximum-clade-credibility tree along with divergence time estimates were visualized and drawn using the program FigTree (http:/ / tree.bio.ed.ac.uk/software/ figtree) (Supplementary Figure S1).

\subsection{Estimation of $C p G$ Content}

The CpG contents were estimated by counting of the number of CG dinucleotides in a genome and divided by the total number of nucleotides.

$$
\text { CpG content }(\%)=\frac{\text { Number of CpG nucleotides }}{\text { Total number of nucleotides }} \times 100
$$

To estimate $\mathrm{CpG}$ content at synonymous positions we included $\mathrm{C}$ in the third codon position followed by $\mathrm{G}$ in the (next) first codon position. Similarly, to calculate $\mathrm{CpG}$ content for nonsynonymous sites, $\mathrm{C}$ in the first codon position followed by $\mathrm{G}$ in the second codon position, and $C$ in the second codon position, and $G$ in the third codon position were included. To calculate the ratio of observed to expected $\mathrm{CpG}$ content $\left(I_{C p G}\right)$, we used the proportion of $C G$ dinucleotides $\left(P_{C p G}\right)$ in the genome divided by the frequency of $C\left(P_{C}\right)$ and $\mathrm{G}\left(P_{G}\right)$ nucleotides [17] as:

$$
I_{C p G}=\frac{P_{C p G}}{P_{C} P_{G}}
$$

\section{Results}

\subsection{Progressive Decline of C $p G$ Dinucleotides in the SARS-CoV-2 Lineage}

The whole genome of SARS-CoV-2 contains $1.47 \%$ of $\mathrm{CpG}$ dinucleotides, which is much less than that observed for other betacoronaviruses. In order to investigate the time of origin of $\mathrm{CpG}$ depletion in the SARS-CoV-2 lineage, we obtained the complete genome sequences of the representatives of all subspecies included in the genus Betacoronavirus. The 79 viral genomes (Supplementary Table S1) were aligned, and a Maximum Likelihood tree was constructed (Figure 1). Each node of the tree was painted with a distinct color to highlight eight distinct phyletic groups. The $\mathrm{CpG}$ content was estimated for each genome and the average $\mathrm{CpG}$ content was estimated for each phyletic group. As shown in Figure 1-inset and Figure 2, the CpG content is the highest for the Nobecovirus genomes (red circles), which progressively declines towards the descending phyletic groups and reaches to the lowest for SARS-CoV-2 and its close relatives (dark blue circles). In contrast, the ancestral groups Merbecovirus and Embecovirus have a lower CpG content than that of the Nobecovirus genomes.

To further confirm this pattern, we estimated the divergence times between SARS-CoV2 and all other betacoronaviruses (Supplementary Figure S1) and plotted them against their respective $\mathrm{CpG}$ contents. Figure 2A shows an increase in the $\mathrm{CpG}$ contents with the increase in the time of divergence of Sarbecovirus, Hibecovirus, and Nobecovirus genomes with SARS$\mathrm{CoV}-2$. However, this trend reverses due to the relatively low genomic $\mathrm{CpG}$ contents of Merbecovirus and Embecovirus. The overall correlation was not significant $(\rho=0.18, p=0.12)$. However, after removing these two groups, the correlation becomes highly significant $(\rho=0.86, p<0.00001)$ (Figure 2B). Looking at this result reveals that the CpG dinucleotides progressively declined in the SARS-CoV-2 lineage over time. For instance, the average CpG content of Nobecovirus genomes was $2.82 \%$, which was almost two times higher than that estimated for SARS-CoV-2 and its close relatives (1.46\%) (Figure 2B). Figure 1 (inset) 
and Figure 2 suggest that the systematic depletion of CpGs might have started in the SARS-CoV-2 lineage after the Hibecovirus + Sarbecovirus clade separated from Nobecovirus (see arrow-Figure 1).

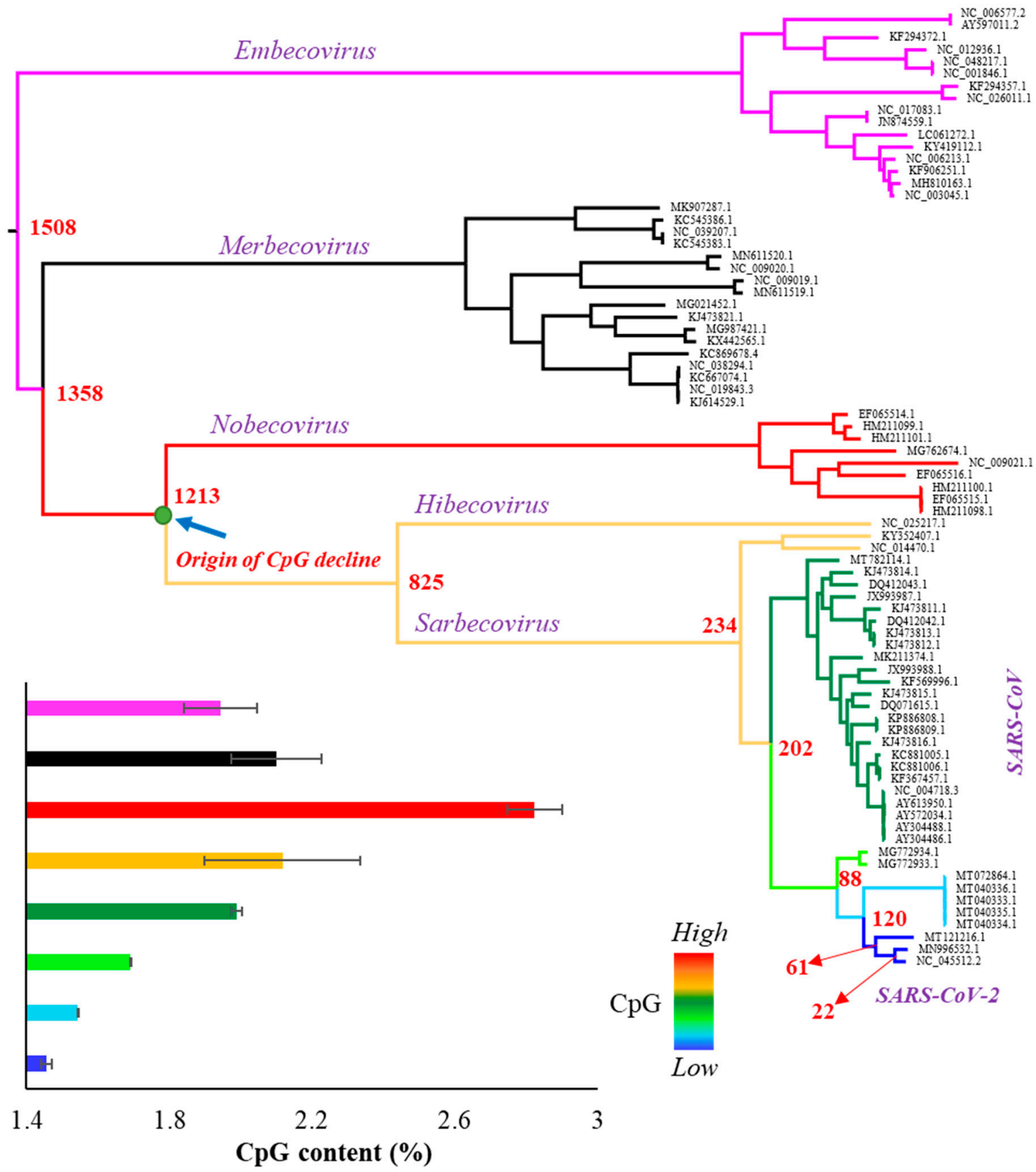

Figure 1. Phylogenetic relationship among 79 representative genomes (Supplementary Table S1) belonging to the genus Betacoronavirus including severe acute respiratory syndrome coronavirus 2 (SARS-CoV-2) (NC_045512.2). The tree was inferred using the maximum likelihood method. Red to blue colors indicate the levels of CpG content: red (2.82\%), orange $(2.12 \%)$, dark green $(1.98 \%)$, pale green $(1.69 \%)$, sky blue $(1.54 \%)$, and dark blue $(1.46 \%)$. Bayesian MCMC method base was used to estimate the time of divergence for each node that are shown in red (Supplementary Figure S1). Inset: column graph showing the $\mathrm{CPG}$ contents (\%) of various clades of the tree. Error bars indicate standard error of the mean. 

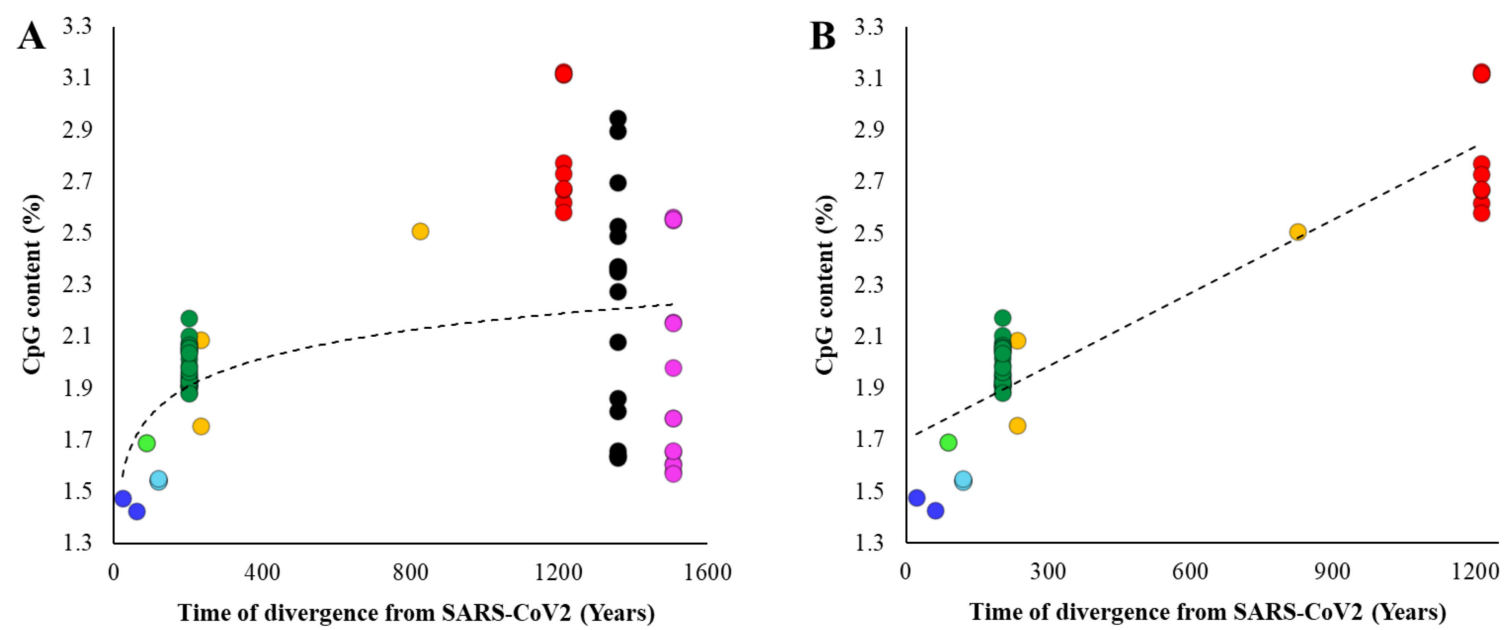

Figure 2. Relationship between divergence times of other betacoronaviruses from SARS-CoV-2 and their CpG contents. The divergence times for each node shown in Figure 1. (A) All genomes were included. The correlation is not significant $(\rho=0.18, p=0.12)$. (B) Embecovirus and Merbecovirus genomes were excluded. The correlation is highly significant $(\rho=0.86$, $p<0.00001)$. Best fitting regression lines are shown.

\subsection{Natural Selection and $C p G$ Deficiency}

The rate of $\mathrm{CpG}$ mutations is well known to be $>10$ times higher than that of the non-CpG nucleotides [29]. Therefore, the decline in the CpG contents could be explained by the process of mutation from $\mathrm{CpG}$ to non-CpG nucleotides. However, $\mathrm{CpG}$ nucleotides are present in functionally important positions of the genome as well. Therefore, mutation of those $\mathrm{CpG}$ dinucleotides will not be tolerated as they are required for the survival of the virus itself and, thus, are expected to be under natural selection [30,31]. To investigate the role of selection, we estimated the $\mathrm{CpG}$ contents of synonymous (neutral-under no selection) and nonsynonymous (under selection) sites of Nobecovirus, Hibecovirus, and Sarbecovirus genomes and plotted them against the times of divergence from SARS-CoV-2. Similar to the genomic correlation shown in Figure 2B, we observed highly significant correlations between the two variables for synonymous as well as nonsynonymous sites (Table 1). The slope of the regression line is almost four times much steeper for synonymous sites compared to that of nonsynonymous positions (Table 1). This is because the magnitude of CpG depletion was much higher for the former compared to the latter. For instance, the average $\mathrm{CpG}$ content of the synonymous sites of SARS-CoV-2 and its close relatives (deep blue in Figure 1) is 2.61 times higher than that estimated for Nobecovirus (red in Figure 1) $(5.9 \%$ vs. $2.0 \%)$. In contrast, this difference was only 1.87 times for nonsynonymous sites ( $2.5 \%$ vs. $1.3 \%)$.

Table 1. Correlation coefficient and significance of relationships with the divergence times between SARS-CoV-2 and other betacoronaviruses.

\begin{tabular}{cccc}
\hline Y-Axis & $\begin{array}{c}\text { Slope of the } \\
\text { Regression Line }\end{array}$ & $\begin{array}{c}\text { Correlation } \\
\text { Coefficient }\end{array}$ & Significance \\
\hline Synonymous positions & 0.0031 & 0.8 & $P<0.00001$ \\
Nonsynonymous positions & 0.0008 & 0.86 & $P<0.00001$ \\
Arginine content & 0.0005 & 0.79 & $P<0.00001$ \\
Proportion of CpG containing & 0.0163 & 0.87 & $P<0.00001$ \\
codons coding for Arginine & & & \\
\hline
\end{tabular}

\subsection{Evolution of Arginine Content and $C p G$}

In order to further confirm the effects of natural selection, we examined the proportion of arginine content. This is because arginine is the only amino acid with codons containing CpG nucleotides in the first two positions (CGA, CGG, CGT, and CGC). However, arginine 
is also coded by two non-CpG codons (AGA and AGG) and, hence, two-thirds (4 out of 6) of the codons contain $\mathrm{CpG}$ dinucleotides in the first two positions. We first estimated the proportion of $\mathrm{CpG}$ containing the codons code for arginine in different betacoronavirus exomes and plotted this against the time of divergence from SARS-CoV-2. A significant positive correlation between the two variables was observed (Table 1). The CpG containing codons coded for $63.7 \%$ of the arginine in the exomes of Nobecovirus (Figure 3A), which is roughly as expected $(2 / 3 \mathrm{rd})$. Whereas this proportion dropped down to $42 \%$ in the exomes of SARS-CoV-2 and its close relatives owing to the loss of CpG dinucleotides in these genomes.
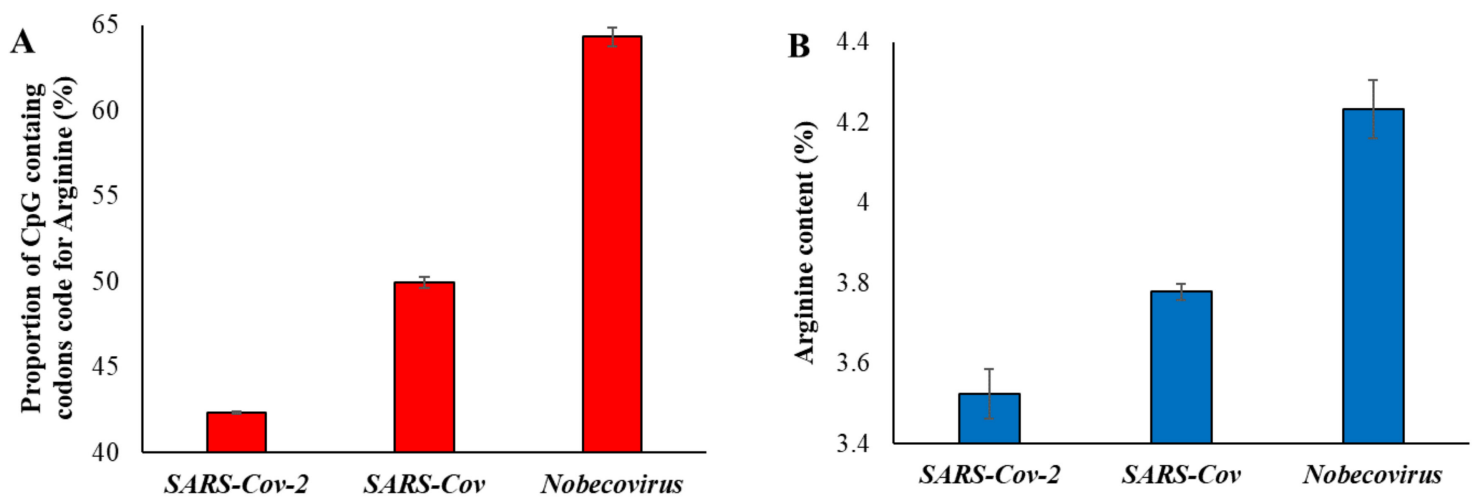

Figure 3. (A) Proportion of $\mathrm{CpG}$ containing the codons code for the amino acid arginine in three groups of virus genomes. (B) Proportion of arginine amino acid present in the exomes of SARS-CoV-2 (and its relatives-dark blue in Figure 1), SARS-CoV (dark green in Figure 1) and Nobecovirus (red in Figure 1). Error bars denote standard error of the mean.

We then estimated the arginine content of the genomes of betacoronaviruses and plotted them against the time of divergence from SARS-CoV-2. This also revealed a significant positive correlation (Table 1). The average arginine content of SARS-CoV-2 and its close relatives was $3.5 \%$, which was significantly less than that observed for Nobecovirus genomes (4.2\%) (Figure 3B).

\subsection{Observed and Expected Proportions of $C p G\left(I_{C p G}\right)$}

Although previous results clearly showed a systematic decline in the $\mathrm{CpG}$ content in the SARS-CoV-2 lineage over time, this could also be due to the reduction in GC contents in the genomes analyzed. To disentangle CpG content from GC content, we estimated the ratio of observed to expected GC content $\left(I_{C p G}\right)$ for the whole viral genomes using equation 2 (see methods). The correlation between $I_{C p G}$ and divergence times (Figure 4) was highly significant $(\rho=0.87, p<0.00001)$ and very similar to that observed for the genomic $\mathrm{CpG}$ content (Figure 2B). The mean $I_{\mathrm{C} p \mathrm{G}}$ estimated for SARS-CoV-2 and its close relative genomes was $63 \%$ smaller than that observed for the Nobecovirus genomes $(0.40 \mathrm{vs}$. 0.66). Similar analysis was performed, and highly significant correlations were observed for synonymous $(\rho=0.88, p<0.00001)$ and nonsynonymous $(\rho=0.88, p<0.00001)$ positions of the exomes of these viruses. The average $I_{C p G}$ estimated for nonsynonymous positions of Nobecovirus exomes was 0.92 , which almost close to the expectation. However, this value was only 0.58 for the exomes of SARS-CoV-2 and its close relatives. On the other hand, the $I_{C P G}$ estimated for synonymous positions was 0.62 for the Nobecovirus exomes and only 0.37 for SARS-CoV-2 and its close relatives. 


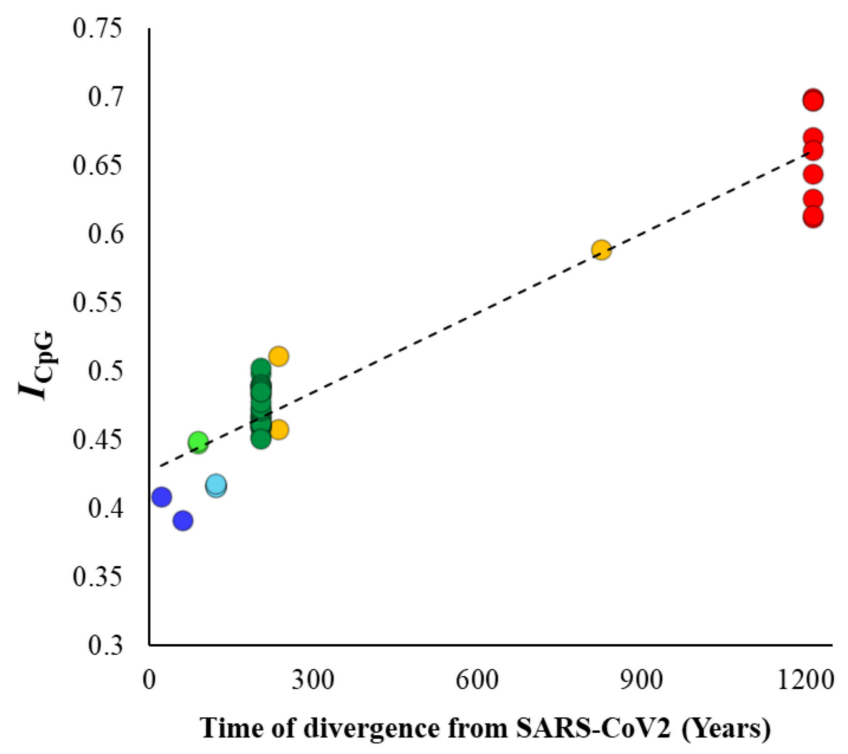

Figure 4. Correlation between the ratio of observed-to-expected $\mathrm{CpG}$ contents $\left(I_{C p G}-\right.$ Equation (2) - see methods) of the betacoronavirus genomes and their respective times of divergence from SARS-CoV-2. The relationship is highly significant $(\rho=0.87, p<0.00001)$.

\section{Discussion}

Although a number of previous studies reported about the reduced CpG content of SARS-CoV-2 genomes $[14,17,18,20]$, the present study showed that the CpG content has been progressively declining in this lineage over a longtime. We showed that this depletion might have started in the Sarbecovirus + Hibecovirus clade after it branched off from the Nobecovirus group (Figure 1). Based on the age of this node it is clear that the CpG content in SARS-CoV-2 lineage has been gradually declining over the past 1213 years. The cause for the reduction could be to evade the antiviral zinc finger protein of the hosts that targets $\mathrm{CpG}$ nucleotides [22,23]. Since the viral genomes belonging to Sarbecovirus + Hibecovirus groups infects many mammals, including human, bat, civet, pangolin, and mink (Supplementary Table S1), the progressive decline suggests that antiviral activities of zinc finger proteins appear to be similar across these mammals. Although $\mathrm{CpG}$ was much lower in the vast majority ( $>90 \%$ ) of the SARS-CoV-2 genome, a few small pockets of the genome consisting of UTRs, envelop and nucleocapsid genes were shown to have relatively high CpGs [32]. Our analysis also showed a relatively higher CpG in these regions (3.5\%) compared to that observed for the whole genome (2.1\%). The combined CpG content of these regions (3.3\%) is still lower for SARS-CoV-2 (and its close relatives) compared to Hibecovirus and Nobecovirus genomes (3.6\%).

Our results showed that the reduction of $\mathrm{CpG}$ nucleotides occur in synonymous and nonsynonymous sites. The reduction in the $\mathrm{CpG}$ content of the latter suggests that not all nonsynonymous $\mathrm{CpG}$ nucleotides are under high selective constraints, and those under less or no selective constraints could have been mutated to non-CpG nucleotides. This is further confirmed by our analysis on arginine contents. We observed a shift in the codons coding for arginine amino acids from $\mathrm{CpG}$ to non-CpG codons in the SARS-CoV-2 lineage over time. However, we also observed a reduction in the proportion of arginine amino acids in this lineage, which suggests that some of these amino acid changes are tolerated in these viruses.

The results of the present study could be useful in designing universal vaccines [33]. For example, the reduction in $\mathrm{CpG}$ content suggests a higher rate of $\mathrm{CpG} \rightarrow \mathrm{UpG}$ mutations. Hence, we can predict that the $\mathrm{CpG}$ nucleotides in genomic regions (e.g., of the $\mathrm{S}$ gene) constituting the epitopes are more likely to be mutated to UpG compared to the mutations in the opposite direction. Therefore, knowledge about the most probable future mutation 
types is immensely useful in designing broad-spectrum epitopes that recognizes many strains of SARS-CoV-2, and its descendants emerging in the imminent future.

\section{Conclusions}

Using all representative genomes of the genus Betacoronavirus, we showed that the CPG dinucleotide has been gradually decreasing in the lineage of SARS-CoV-2 in the past 1213 years since it separated from Nobecovirus. Analyses on protein-coding exomes revealed that $\mathrm{CpG}$ reduction occurs at neutrally evolving - as well as selectively constrainedpositions. Our results on the ratio of observed to expected CpG content clearly showed that the reduction in $\mathrm{CpG}$ content is not due to the reduction in the GC contents of the genome. These results are not only useful in understanding the evolution of SARS-CoV-2, but also in developing vaccines that are effective on current-as well as future-strains of COVID-19.

Supplementary Materials: The following are available online at https://www.mdpi.com/2079-7 $737 / 10 / 1 / 52 / s 1$, Table S1: list of betacoronavirus genomes used in the study. Figure S1: Bayesian MCMC time tree. Divergence times are given on the nodes.

Funding: This research was funded by the University of the Sunshine Coast (DVC-R\&I grant).

Institutional Review Board Statement: Not applicable.

Informed Consent Statement: Not applicable.

Data Availability Statement: No new data were created in this study. Data sharing is not applicable to this article.

Conflicts of Interest: The author declares no conflict of interest.

\section{References}

1. Bchetnia, M.; Girard, C.; Duchaine, C.; Laprise, C. The outbreak of the novel severe acute respiratory syndrome coronavirus 2 (SARS-CoV-2): A review of the current global status. J. Infect Public Health 2020, 13, 1601-1610. [CrossRef]

2. Boni, M.F.; Lemey, P.; Jiang, X.; Lam, T.T.; Perry, B.W.; Castoe, T.A.; Rambaut, A.; Robertson, D.L. Evolutionary origins of the SARS-CoV-2 sarbecovirus lineage responsible for the COVID-19 pandemic. Nat. Microbiol. 2020, 5, 1408-1417. [CrossRef]

3. Coronaviridae Study Group of the International Committee on Taxonomy of Viruses. The species Severe acute respiratory syndrome-related coronavirus: Classifying 2019-nCoV and naming it SARS-CoV-2. Nat. Microbiol. 2020, 5, 536-544. [CrossRef]

4. Lu, R.; Zhao, X.; Li, J.; Niu, P.; Yang, B.; Wu, H.; Wang, W.; Song, H.; Huang, B.; Zhu, N.; et al. Genomic characterisation and epidemiology of 2019 novel coronavirus: Implications for virus origins and receptor binding. Lancet 2020, 395, 565-574. [CrossRef]

5. Mercatelli, D.; Giorgi, F.M. Geographic and Genomic Distribution of SARS-CoV-2 Mutations. Front. Microbiol. 2020, 11, 1800. [CrossRef]

6. V'Kovski, P.; Kratzel, A.; Steiner, S.; Stalder, H.; Thiel, V. Coronavirus biology and replication: Implications for SARS-CoV-2. Nat. Rev. Microbiol. 2020. [CrossRef]

7. Worobey, M.; Pekar, J.; Larsen, B.B.; Nelson, M.I.; Hill, V.; Joy, J.B.; Rambaut, A.; Suchard, M.A.; Wertheim, J.O.; Lemey, P. The emergence of SARS-CoV-2 in Europe and North America. Science 2020, 370, 564-570. [CrossRef] [PubMed]

8. Zhang, L.P.; Wang, M.; Wang, Y.; Zhu, J.; Zhang, N. Focus on the 2019 novel coronavirus (SARS-CoV-2). Future Microbiol. 2020, 15, 905-918. [CrossRef] [PubMed]

9. Berkhout, B.; van Hemert, F. On the biased nucleotide composition of the human coronavirus RNA genome. Virus. Res. 2015, 202, 41-47. [CrossRef] [PubMed]

10. Digard, P.; Lee, H.M.; Sharp, C.; Grey, F.; Gaunt, E. Intra-genome variability in the dinucleotide composition of SARS-CoV-2. Virus. Evol. 2020, 6, veaa057. [CrossRef] [PubMed]

11. Grigoriev, A. Mutational patterns correlate with genome organization in SARS and other coronaviruses. Trends Genet. 2004, 20, 131-135. [CrossRef] [PubMed]

12. Khrustalev, V.V.; Giri, R.; Khrustaleva, T.A.; Kapuganti, S.K.; Stojarov, A.N.; Poboinev, V.V. Translation-Associated Mutational U-Pressure in the First ORF of SARS-CoV-2 and Other Coronaviruses. Front. Microbiol. 2020, 11, 559165. [CrossRef] [PubMed]

13. Pyrc, K.; Jebbink, M.F.; Berkhout, B.; van der Hoek, L. Genome structure and transcriptional regulation of human coronavirus NL63. Virol. J. 2004, 1, 7. [CrossRef] [PubMed]

14. Rice, A.M.; Morales, A.C.; Ho, A.T.; Mordstein, C.; Muhlhausen, S.; Watson, S.; Cano, L.; Young, B.; Kudla, G.; Hurst, L.D. Evidence for strong mutation bias towards, and selection against, U content in SARS-CoV-2: Implications for vaccine design. Mol. Biol. Evol. 2020. [CrossRef] [PubMed] 
15. Simmonds, P. Rampant C->U Hypermutation in the Genomes of SARS-CoV-2 and Other Coronaviruses: Causes and Consequences for Their Short- and Long-Term Evolutionary Trajectories. mSphere 2020, 5. [CrossRef]

16. Tort, F.L.; Castells, M.; Cristina, J. A comprehensive analysis of genome composition and codon usage patterns of emerging coronaviruses. Virus. Res. 2020, 283, 197976. [CrossRef]

17. Xia, X. Extreme Genomic CpG Deficiency in SARS-CoV-2 and Evasion of Host Antiviral Defense. Mol. Biol. Evol. 2020, 37, 2699-2705. [CrossRef]

18. Pollock, D.D.; Castoe, T.A.; Perry, B.W.; Lytras, S.; Wade, K.J.; Robertson, D.L.; Holmes, E.C.; Boni, M.F.; Kosakovsky Pond, S.L.; Parry, R.; et al. Viral CpG Deficiency Provides No Evidence That Dogs Were Intermediate Hosts for SARS-CoV-2. Mol. Biol. Evol. 2020, 37, 2706-2710. [CrossRef]

19. Vacca, I. Viral infection: Adapt or get zapped. Nat. Rev. Microbiol. 2017, 15, 641. [CrossRef]

20. Wang, Y.; Mao, J.M.; Wang, G.D.; Luo, Z.P.; Yang, L.; Yao, Q.; Chen, K.P. Human SARS-CoV-2 has evolved to reduce CG dinucleotide in its open reading frames. Sci. Rep. 2020, 10, 12331. [CrossRef]

21. Ficarelli, M.; Antzin-Anduetza, I.; Hugh-White, R.; Firth, A.E.; Sertkaya, H.; Wilson, H.; Neil, S.J.D.; Schulz, R.; Swanson, C.M. CpG Dinucleotides Inhibit HIV-1 Replication through Zinc Finger Antiviral Protein (ZAP)-Dependent and -Independent Mechanisms. J. Virol. 2020, 94. [CrossRef] [PubMed]

22. Luo, X.; Wang, X.; Gao, Y.; Zhu, J.; Liu, S.; Gao, G.; Gao, P. Molecular Mechanism of RNA Recognition by Zinc-Finger Antiviral Protein. Cell Rep. 2020, 30, 46-52. [CrossRef] [PubMed]

23. Meagher, J.L.; Takata, M.; Goncalves-Carneiro, D.; Keane, S.C.; Rebendenne, A.; Ong, H.; Orr, V.K.; MacDonald, M.R.; Stuckey, J.A.; Bieniasz, P.D.; et al. Structure of the zinc-finger antiviral protein in complex with RNA reveals a mechanism for selective targeting of CG-rich viral sequences. Proc. Natl. Acad. Sci. USA 2019, 116, 24303-24309. [CrossRef] [PubMed]

24. Stamatakis, A. RAxML version 8: A tool for phylogenetic analysis and post-analysis of large phylogenies. Bioinformatics 2014, 30, 1312-1313. [CrossRef]

25. Kumar, S.; Stecher, G.; Li, M.; Knyaz, C.; Tamura, K. MEGA X: Molecular Evolutionary Genetics Analysis across Computing Platforms. Mol. Biol. Evol. 2018, 35, 1547-1549. [CrossRef]

26. Bouckaert, R.; Vaughan, T.G.; Barido-Sottani, J.; Duchene, S.; Fourment, M.; Gavryushkina, A.; Heled, J.; Jones, G.; Kuhnert, D.; De Maio, N.; et al. BEAST 2.5: An advanced software platform for Bayesian evolutionary analysis. PLoS Comput. Biol. 2019, 15, e1006650. [CrossRef]

27. Duchene, S.; Featherstone, L.; Haritopoulou-Sinanidou, M.; Rambaut, A.; Lemey, P.; Baele, G. Temporal signal and the phylodynamic threshold of SARS-CoV-2. Virus Evol. 2020, 6, veaa061. [CrossRef]

28. Rambaut, A.; Drummond, A.J.; Xie, D.; Baele, G.; Suchard, M.A. Posterior Summarization in Bayesian Phylogenetics Using Tracer 1.7. Syst. Biol. 2018, 67, 901-904. [CrossRef]

29. Subramanian, S.; Kumar, S. Neutral substitutions occur at a faster rate in exons than in noncoding DNA in primate genomes. Genome Res. 2003, 13, 838-844. [CrossRef]

30. Li, W.-H. Molecular Evolution; Sinauer Associates: Sunderland, MA, USA, 1997.

31. Subramanian, S.; Kumar, S. Higher intensity of purifying selection on $>90 \%$ of the human genes revealed by the intrinsic replacement mutation rates. Mol. Biol. Evol. 2006, 23, 2283-2287. [CrossRef]

32. Nchioua, R.; Kmiec, D.; Muller, J.A.; Conzelmann, C.; Gross, R.; Swanson, C.M.; Neil, S.J.D.; Stenger, S.; Sauter, D.; Munch, J.; et al. SARS-CoV-2 Is Restricted by Zinc Finger Antiviral Protein despite Preadaptation to the Low-CpG Environment in Humans. $m$ Bio 2020, 11. [CrossRef] [PubMed]

33. Giurgea, L.T.; Han, A.; Memoli, M.J. Universal coronavirus vaccines: The time to start is now. NPJ Vaccines 2020, 5, 43. [CrossRef] [PubMed] 